\title{
Etiología de la neumonía bacteriana adquirida en la comunidad en niños hospitalizados. Uruguay 1998-2004
}

\author{
Ana María Ferrari C., M. Catalina Pirez G., Adriana Martínez A., Gabriela Algorta R., Flavia Chamorro V., \\ M. José Guala B., Cristina Zabala Ch., Gustavo Giachetto L. y Alicia Montano L.
}

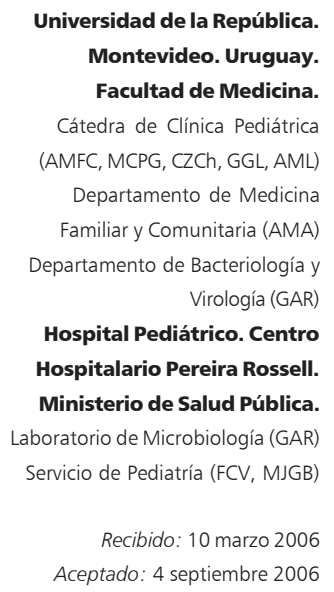

Universidad de la República. Montevideo. Uruguay. Facultad de Medicina. Cátedra de Clínica Pediátrica (AMFC, MCPG, CZCh, GGL, AML) Departamento de Medicina Familiar y Comunitaria (AMA) Departamento de Bacteriología y Virología (GAR) Hospital Pediátrico. Centro Hospitalario Pereira Rossell. Ministerio de Salud Pública. Laboratorio de Microbiología (GAR) Servicio de Pediatría (FCV, MJGB)

Recibido: 10 marzo 2006 Aceptado: 4 septiembre 2006

Correspondencia a: Ana María Ferrari Castilla aferrari@chasque.apc.org

\section{Etiology of community acquired pneumonia in inpatients children. Uruguay 1998-2004}

Community acquired bacterial pneumonia represents $8.7 \%$ of overall discharges of children under 15 years old in the Pediatric Hospital of the Centro Hospitalario Pereira Rossell; since 1997 treatment guidelines are being applied. Changes in etiology of this disease in children hospitalized in our institution from 1998 to 2004 were studied. Streptococcus pneumoniae was the most frequent agent in every year of the period, $91.4 \pm 2.3 \%$; its susceptibility to penicillin has remained stable; serotypes 14,1 and 5 were the most frequent isolated. In 2002 the first case of community acquired methicillin resistant S. aureus was identified; isolations of this microorganism were 5.8\% in 2003 and $6.3 \%$ in 2004. Surveillance of etiology of community acquired pneumonia is mandatory in order to adjust guidelines to changes in agents and their antimicrobial susceptibility.

Key words: Pneumonia, Etiology, children, Streptococcus pneumoniae, Staphylococcus aureus.

Palabras claves: Neumonía, Etiología, niños, Streptococcus pneumoniae, Staphylococcus aureus.

\section{Introducción}

$\mathrm{L}$ a neumonía bacteriana adquirida en la comunidad (NBAC) representa en promedio $8,7 \%$ del total de egresos hospitalarios de niños bajo 15 años de edad asistidos en el Hospital Pediátrico del Centro Hospitalario Pereira Rossell (CHPR) desde el año 2000. A partir de 1997 se aplican guías de diagnóstico y tratamiento de esta patología ${ }^{1}$, cuyo cumplimiento y eficacia son evaluados periódicamente ${ }^{2,3}$. Las recomendaciones para el tratamiento antimicrobiano se basan en el conocimiento de los agentes etiológicos de neumonía y de su susceptibilidad in vitro a nivel local. Se realiza la vigilancia continua de los agentes bacterianos de neumonía adquirida en la comunidad a fin de ajustar dichas recomendaciones a los cambios que puedan producirse, tanto en los agentes como en su susceptibilidad antimicrobiana. Esta vigilancia permite además realizar recomendaciones sobre medidas preventivas. El mejor ejemplo lo constituye la incorporación de la vacuna anti Haemophilus influenzae tipo b en el año 1994, que determinó una reducción significativa de las neumonías producidas por esta bacteria ${ }^{4-6}$. En el momento actual, los principales problemas se refieren a la necesidad de una vacuna anti-neumo- cóccica que confiera protección efectiva contra los serotipos locales y a la emergencia de una cepa de Staphylococcus aureus resistente a meticilina con características especiales de resistencia y de patogenicidad, conocida como S. aureus meticilina-resistente adquirido en la comunidad (SAMR-AC) $)^{7}$. En algunos países la vacunación con vacuna anti-neumocóccica conjugada de siete serotipos $(4,6 \mathrm{~B}, 9 \mathrm{~V}, 14,18 \mathrm{C}, 19 \mathrm{~F}$, 23F) a niños bajo cinco años de edad, ha sido exitosa en la disminución de la frecuencia de enfermedades neumocóccicas, incluida la neumonía ${ }^{8,9}$. La información disponible en nuestro país muestra que los serotipos 14,1 y 5 son los más frecuentemente asociados a neumonía ${ }^{10}$. El desarrollo de vacunas conjugadas efectivas contra una gama más amplia de serotipos o de vacunas no polisacarídicas puede ser la respuesta a este problema. La aparición de SAMR-AC como agente de neumonía constituye un desafío clínico y terapéutico en diversas regiones del mundo y requiere estrategias específicas para su abordaje.

Objetivo. Describir la etiología de la neumonía bacteriana adquirida en la comunidad (NBAC) en niños hospitalizados en el Hospital Pediátrico del Centro Hospitalario Pereira Rossell (HP-CHPR) observada en el período 1998-2004. 
De acuerdo con las pautas de diagnóstico, tratamiento y prevención del hospital ${ }^{11}$, neumonía se define como: presencia de polipnea y/o tiraje en un niño con tos y se considera de etiología bacteriana por el hallazgo radiológico de consolidación parenquimatosa y/o efusión pleural. La etiología se confirma por la realización de hemocultivo y de cultivo del líquido pleural obtenido por toracocentesis cuando corresponde. Se realiza detección de antígenos capsulares por técnica de látex en líquido pleural cuando no se aísla un microorganismo y si se dispone del reactivo correspondiente.

Los cultivos, la identificación del agente aislado y la susceptibilidad a los antimicrobianos se realizaron por técnicas microbiológicas habituales; se determinó CIM para S. pneumoniae por eliptograma-E-test $\mathrm{t}^{12}$. Se consideraron sensibles a penicilina las cepas con CIM $\leq 0,06 \mu \mathrm{g} / \mathrm{ml}$, con susceptibilidad intermedia las con CIM de $0,12-1,0 \mu \mathrm{g} / \mathrm{ml}$ y resistentes con CIM $\geq 2,0 \mu \mathrm{g} /$ $\mathrm{ml}$. Se consideraron sensibles a cefotaxima las cepas con $\mathrm{CIM} \leq 0,5 \mu \mathrm{g} / \mathrm{ml}$, con susceptibilidad intermedia las con $\mathrm{CIM}=1,0 \mu \mathrm{g} / \mathrm{ml}$ y resistentes $\operatorname{con} \mathrm{CIM} \geq 2,0$ $\mu \mathrm{g} / \mathrm{ml}^{13}$.

Para el análisis estadístico de los datos se utilizó el programa estadístico Epi-Info (versión 6.0; CDC, Atlanta, GA). Para la comparación de porcentajes se aplicó el test de $\chi^{2}$ y el test de Fisher para valores menores a 5 ; se consideró significativo $\mathrm{p}<0,05$. Los datos de susceptibilidad a los antimicrobianos se expresan como promedios y su intervalo de confianza de 95\% (IC 95\%).

\section{Resultados}

La frecuencia anual de los casos de NBAC en el período 1998-2004 se muestra en la Tabla 1. A partir del año 2000 se dispone de información confiable sobre el número de niños con NBAC asistidos en el HP-CHPR. En los años 1998 y 1999 las neumonías se registraban sin tener en cuenta su probable etiología. Si excluimos estos dos años, el promedio anual de egresos por neumonía fue 1.126. En el total de egresos del período las neumonías representaron en promedio $8,73 \%$ (rango $7,62-9,85 \%)$.

La etiología bacteriana en el período 2000-2004 se confirmó en 461 niños que representan $8,18 \%$ de los casos con diagnóstico de NBAC (Tabla 2). El diagnóstico microbiológico ha aumentado en los dos últimos años.

En los 7 años (1998-2004) se identificaron en sangre y/o líquido pleural de 555 niños, 560 cepas bacterianas. Streptococcus pneumoniae fue el agente más frecuente en todos los años del período, 91,4 $\pm 2,3 \%$. En el año 2002 se identificó el primer caso de NBAC causada por

\section{Material y Métodos}

Se incluyeron en el estudio todos los microorganismos identificados en niños hospitalizados con NBAC entre el 1\%enero/1998 y el 31/diciembre/2004.

Los datos sobre características de estos microorganismos y su susceptibilidad a los antimicrobianos se obtuvieron del sistema de registro informatizado del Laboratorio de Microbiología del CHPR.

La serotipificación de $S$. pneumoniae fue realizada en el Laboratorio Central de Salud Pública*.

Los datos referidos a los pacientes se obtuvieron del sistema de registro informatizado del HP del CHPR y de las historias clínicas de los niños.

Tabla 1. Neumonía bacteriana adquirida en la comunidad. Egresos anuales 1998-2004. Hospital Pediátrico. Centro Hospitalario Pereira Rossell

\begin{tabular}{|lccc|} 
Año & No & total de egresos & Neumonía \\
$\mathbf{n}$ & $\begin{array}{c}\text { bacteriana } \\
\%\end{array}$ \\
\hline 1998 & 10.520 & $2.009 *$ & - \\
1999 & 10.378 & $1.725 *$ & - \\
\hline 2000 & 11.489 & 976 & 8,49 \\
\hline 2001 & 12.018 & 1.119 & 9,31 \\
\hline 2002 & 12.039 & 1.186 & 9,85 \\
\hline 2003 & 13.531 & 1.032 & 7,62 \\
\hline 2004 & 15.413 & 1.318 & 8,55 \\
\hline * Incluye neumonías bacterianas y virales. & & \\
\hline
\end{tabular}

Tabla 2. Neumonía bacteriana adquirida en la comunidad, con etiología confirmada. Hospital Pediátrico. Centro Hospitalario Pereira Rossell 1998-2004

\begin{tabular}{|cccc|} 
Año & $\begin{array}{c}\text { Neumonía probablemente } \\
\text { bacteriana } \\
\mathbf{n}\end{array}$ & \multicolumn{2}{c|}{$\begin{array}{c}\text { Neumonía bacteriana } \\
\text { confirmada } \\
\mathbf{n}\end{array}$} \\
\hline 1998 & - & 33 & - \\
1999 & - & 61 & - \\
\hline 2000 & 976 & 69 & 7,06 \\
\hline 2001 & 1.119 & 60 & 5,36 \\
\hline 2002 & 1.186 & 46 & 3,7 \\
\hline 2003 & 1.032 & 108 & 10,46 \\
\hline 2004 & 1.318 & 178 & 13,50 \\
\hline
\end{tabular}

* Camou T, Palacio R, Hortal M. Laboratorio Central de Salud Pública. Montevideo. Uruguay. Programa OPS/SIREVA. 


\begin{tabular}{|c|c|c|c|c|c|c|}
\hline \multirow[t]{2}{*}{ Año } & \multicolumn{2}{|c|}{ S. pneumoniae } & \multicolumn{2}{|c|}{ S. aureus } & \multirow{2}{*}{$\begin{array}{c}\text { H. influenzae* } \\
\mathbf{n}\end{array}$} & \multirow{2}{*}{$\begin{array}{c}\text { S. pyogenes } \\
\mathbf{n}\end{array}$} \\
\hline & $\mathbf{n}$ & $\%$ & & $\mathbf{n}$ & & \\
\hline 1998 & 30 & 90,9 & SAMS* * & 3 & - & - \\
\hline 1999 & 60 & 96,7 & - & & 2 & - \\
\hline 2000 & 65 & 92,8 & SAMS & 2 & 1 & 2 \\
\hline 2001 & 57 & 94,4 & - & & 3 & - \\
\hline 2002 & 43 & 93,4 & $\begin{array}{l}\text { SAMS } \\
\text { SAMR-AC*** }\end{array}$ & $\begin{array}{l}1 \\
1\end{array}$ & 1 & - \\
\hline 2003 & 92 & 85,1 & $\begin{array}{l}\text { SAMS } \\
\text { SAMR-AC }\end{array}$ & $\begin{array}{l}3 \\
6\end{array}$ & 6 & 1 \\
\hline 2004 & 165 & 91,2 & SAMR-AC & 9 & 6 & 1 \\
\hline Total & 512 & 91,4 & & 25 & 19 & 4 \\
\hline $\begin{array}{l}\text { * } 5 \text { as } \\
\text { * Sta } \\
\text { *** St }\end{array}$ & $\begin{array}{l}\text { pneun } \\
\text { aureus } \\
\text { s aureu }\end{array}$ & sensib & en comunidad & & & \\
\hline
\end{tabular}

Tabla 4. Neumonía adquirida en la comunidad. Tipo de muestra que permitió establecer como agente causal a Streptococcus pneumoniae. Hospital Pediátrico. Centro Hospitalario Pereira Rossell

\begin{tabular}{|c|c|c|c|c|c|}
\hline Año & Hemocultivo & $\begin{array}{l}\text { Cultivo de líquido } \\
\text { pleural }\end{array}$ & $\begin{array}{c}\text { Hemocultivo y cultivo } \\
\text { de líquido pleural }\end{array}$ & $\begin{array}{l}\text { Antígenos capsulares } \\
\text { en líquido pleural }\end{array}$ & Total \\
\hline 1998 & 13 & 16 & 1 & - & 30 \\
\hline 1999 & 33 & 23 & 4 & - & 60 \\
\hline 2000 & 39 & 17 & 9 & - & 65 \\
\hline 2001 & 30 & 24 & 3 & - & 57 \\
\hline 2002 & 17 & 20 & 6 & - & 43 \\
\hline 2003 & 53 & 28 & 3 & 8 & 92 \\
\hline 2004 & 70 & 48 & 12 & 35 & 165 \\
\hline Total & 255 & 176 & 38 & 43 & 512 \\
\hline
\end{tabular}

SAMR-AC, no multi-resistente. De los 19 aislados de H. influenzae, sólo 3 correspondieron al tipo b; 5 se presentaron como co-infección con $S$. pneumoniae (Tabla 3).

El tipo de muestra donde se recuperó $S$. pneumoniae se observa en la Tabla 4. En algunos años la disponibilidad de métodos para detectar antígenos capsulares en líquido pleural permitió diagnosticar un mayor número de casos de neumonía neumocóccica.

Los serotipos predominantes de $S$. pneumoniae fueron: 14,5 y 1 , seguidos por serotipo 3 (Tabla 5); en conjunto estos cuatro serotipos correspondieron a $77 \%$ de las cepas tipificadas en este período.
La susceptibilidad de $S$. pneumoniae a penicilina y cefotaxima se muestra en las Figuras 1 y 2 . Se destaca el aumento en el último año del porcentaje de aislados susceptibles a penicilina $(76 \pm 7,5 \%)$.

Los serotipos 3, 1 y 5 fueron significativamente más frecuentes en los niños con empiema (Tabla 6).

La relación entre serotipo y susceptibilidad a penicilina y a cefotaxima se muestra en las Tabla 7 y 8 . Las cepas resistentes correspondieron exclusivamente al serotipo 14, así como la mayoría de las de resistencia intermedia, tanto para penicilina como para cefotaxima.

De las 19 cepas de Haemophilus sp sólo 3 eran productoras de $\beta$-lactamasas. 


\begin{tabular}{|c|c|c|c|c|c|c|c|c|c|}
\hline Serotipos & $\begin{array}{c}1998 \\
\text { (n: 23) }\end{array}$ & $\begin{array}{c}1999 \\
(n: 43)\end{array}$ & $\begin{array}{c}2000 \\
\text { (n: 65) }\end{array}$ & $\begin{array}{c}2001 \\
\text { (n: } 53)\end{array}$ & $\begin{array}{c}2002 \\
(n: 31)\end{array}$ & $\begin{array}{c}2003 \\
\text { (n: 82) }\end{array}$ & $\begin{array}{c}2004 \\
\text { (n: 119) }\end{array}$ & $\begin{array}{l}\text { Tc } \\
\text { (n: } \\
n\end{array}$ & $\begin{array}{l}\text { tal } \\
\text { 116) }\end{array}$ \\
\hline 14 & 7 & 10 & 20 & 17 & 12 & 36 & 28 & 130 & 31,2 \\
\hline 5 & 2 & 12 & 17 & 5 & 5 & 8 & 34 & 83 & 19,9 \\
\hline 1 & 9 & 8 & 15 & 13 & 7 & 8 & 22 & 82 & 19,7 \\
\hline 3 & 2 & 2 & 3 & 5 & 2 & 4 & 9 & 27 & 6,5 \\
\hline $9 \mathrm{~V}$ & 1 & 0 & 0 & 3 & 0 & 6 & 8 & 18 & 4,3 \\
\hline $7 F$ & 0 & 2 & 3 & 3 & 0 & 5 & 5 & 18 & 4,3 \\
\hline $6 \mathrm{~B}$ & 0 & 3 & 0 & 2 & 1 & 5 & 5 & 16 & 3,8 \\
\hline $19 \mathrm{~A}$ & 0 & 2 & 1 & 1 & 1 & 5 & 3 & 13 & 3,1 \\
\hline $19 \mathrm{~F}$ & 0 & 0 & 2 & 0 & 0 & 1 & 1 & 4 & 0,9 \\
\hline $6 \mathrm{~A}$ & 0 & 0 & 2 & 0 & 0 & 1 & 1 & 4 & 0,9 \\
\hline $12^{*}$ & 0 & 0 & 1 & 2 & 0 & 0 & 0 & 3 & 0,7 \\
\hline 8 & 1 & 0 & 0 & 0 & 0 & 1 & 1 & 3 & 0,7 \\
\hline $18 B$ & 0 & 1 & 1 & 0 & 0 & 0 & 0 & 2 & 0,4 \\
\hline $9 \mathrm{~N}$ & 0 & 2 & 0 & 0 & 0 & 0 & 0 & 2 & 0,4 \\
\hline 4 & 0 & 0 & 0 & 0 & 2 & 0 & 0 & 2 & 0,4 \\
\hline 7 & 1 & 0 & 0 & 0 & 0 & 0 & 0 & 1 & 0,2 \\
\hline 34 & 0 & 1 & 0 & 0 & 0 & 0 & 0 & 1 & 0,2 \\
\hline $23 B$ & 0 & 0 & 0 & 1 & 0 & 0 & 0 & 1 & 0,2 \\
\hline $10 \mathrm{~A}$ & 0 & 0 & 0 & 1 & 0 & 0 & 0 & 1 & 0,2 \\
\hline $33^{*}$ & 0 & 0 & 0 & 0 & 1 & 0 & 0 & 1 & 0,2 \\
\hline 22 & 0 & 0 & 0 & 0 & 0 & 1 & 0 & 1 & 0,2 \\
\hline $23 \mathrm{~F}$ & 0 & 0 & 0 & 0 & 0 & 1 & 0 & 1 & 0,2 \\
\hline 15 & 0 & 0 & 0 & 0 & 0 & 0 & 1 & 1 & 0,2 \\
\hline $18 \mathrm{C}$ & 0 & 0 & 0 & 0 & 0 & 0 & 1 & 1 & 0,2 \\
\hline
\end{tabular}

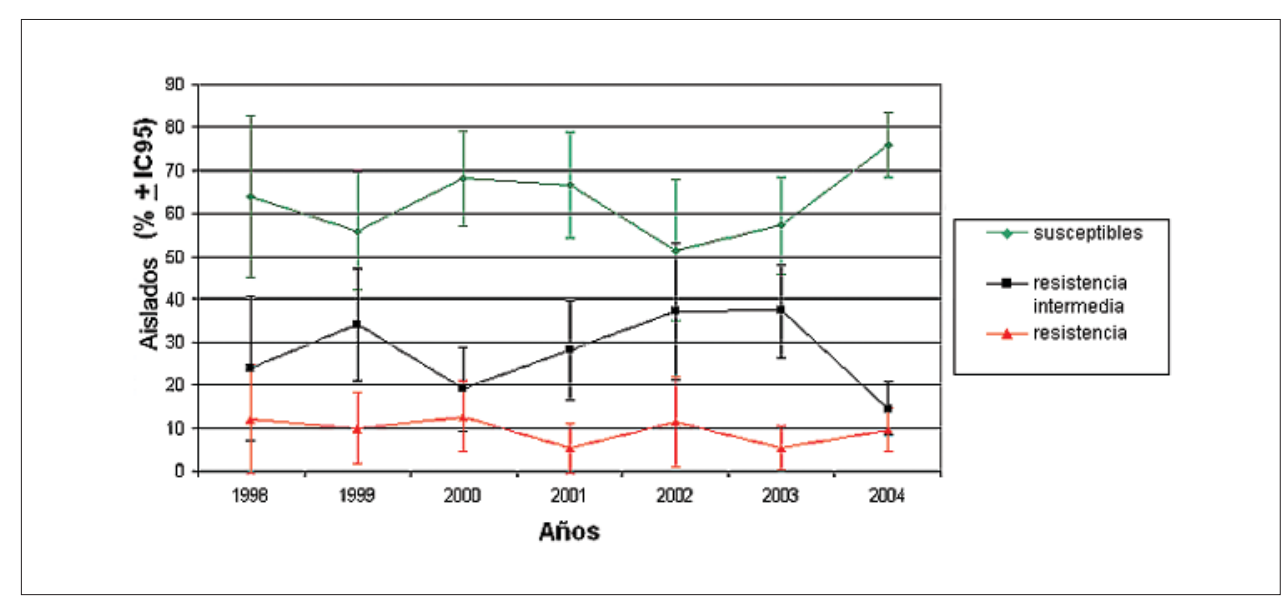

Figura 1. Evolución de la susceptibilidad de Streptococcus pneumoniae a penicilina. Se observan los porcentajes de aislados susceptibles y con resistencia intermedia y absoluta, y sus respectivos intervalos de confianza de 95\%. 
Figura 2. Evolución de la susceptibilidad de Streptococcus pneumoniae a cefotaxima. Se observan los porcentajes de aislados susceptibles y con resistencia intermedia y absoluta, y sus respectivos intervalos de confianza de $95 \%$.

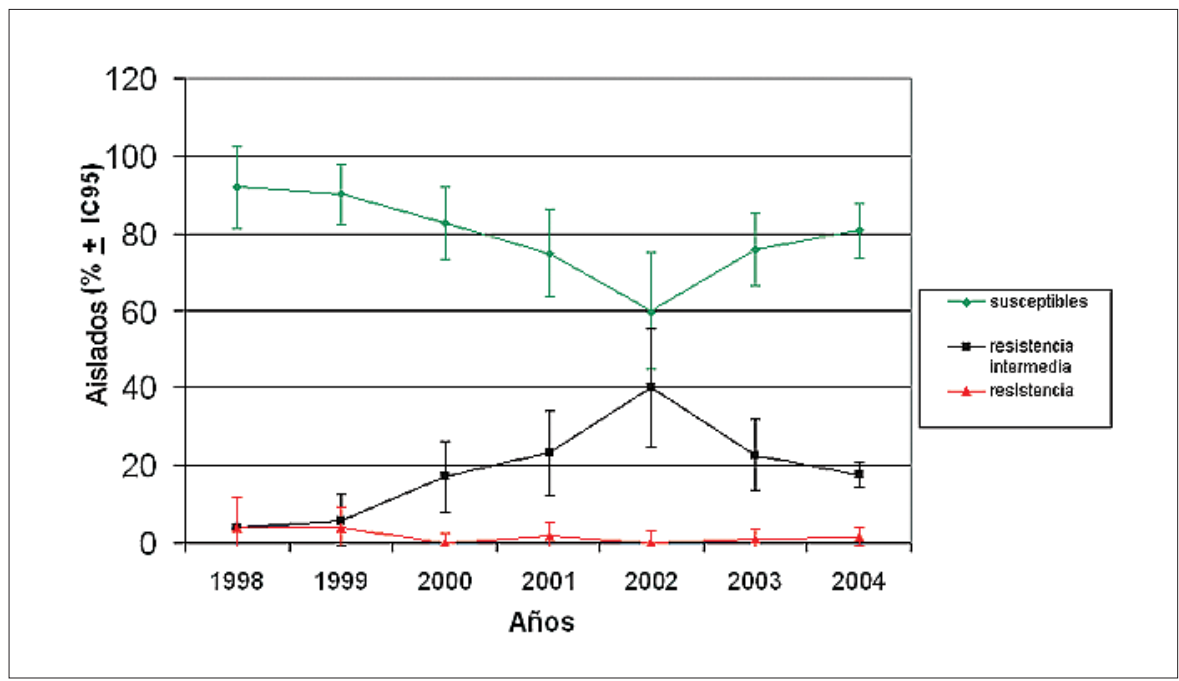

\section{Tabla 6. Serotipos de Streptococcus pneumoniae recuperados} en neumonías con empiema

\begin{tabular}{|c|c|c|c|c|c|}
\hline \multirow[t]{3}{*}{ Serotipo } & \multicolumn{3}{|c|}{ Empiema } & \multirow{3}{*}{$\begin{array}{c}\text { Total } \\
\mathbf{n}\end{array}$} & \multirow[t]{3}{*}{$\mathbf{P}$} \\
\hline & & Sí & No & & \\
\hline & $\mathbf{n}$ & (\%) & n $\quad(\%)$ & & \\
\hline 14 & 57 & $(43,8)$ & $73(56,1)$ & 130 & 0,04 \\
\hline 5 & 55 & $(66,2)$ & $28(33,7)$ & 83 & $<0,01$ \\
\hline 1 & 56 & $(68,2)$ & $26(31,7)$ & 82 & $<0,01$ \\
\hline 3 & 24 & $(88,8)$ & $3(11,1)$ & 27 & $<0,01$ \\
\hline $9 \mathrm{~V}$ & 9 & $(50,0)$ & $9(50,0)$ & 18 & NS \\
\hline $7 F$ & 8 & $(44,4)$ & $10(55,5)$ & 18 & NS \\
\hline $6 B$ & 5 & $(31,2)$ & $11(68,7)$ & 16 & 0,03 \\
\hline $19 A$ & 7 & $(53,8)$ & $6(46,1)$ & 13 & NS \\
\hline $19 \mathrm{~F}$ & & 2 & 2 & 4 & - \\
\hline $6 \mathrm{~A}$ & & 2 & 2 & 4 & - \\
\hline $12^{*}$ & & 1 & 2 & 3 & - \\
\hline 8 & & 2 & 1 & 3 & - \\
\hline $18 \mathrm{~B}$ & & 1 & 1 & 2 & - \\
\hline $9 \mathrm{~N}$ & & 2 & 0 & 2 & - \\
\hline 4 & & 1 & 1 & 2 & - \\
\hline 7 & & 1 & 0 & 1 & - \\
\hline 34 & & 0 & 1 & 1 & - \\
\hline $23 B$ & & 0 & 1 & 1 & - \\
\hline $10 \mathrm{~A}$ & & 1 & 0 & 1 & - \\
\hline $33^{*}$ & & 1 & 0 & 1 & - \\
\hline 22 & & 1 & 0 & 1 & - \\
\hline $23 \mathrm{~F}$ & & 0 & 1 & 1 & - \\
\hline 15 & & 1 & 0 & 1 & - \\
\hline $18 C$ & & 0 & 1 & 1 & - \\
\hline Total & & 237 & 179 & 416 & - \\
\hline
\end{tabular}

No se aplicó test estadístico para comparar los valores menores a 3, dado que la distribución de los mismos es similar
Tabla 7. Susceptibilidad in vitro* a penicilina según serotipo de Streptococcus pneumoniae

\begin{tabular}{|c|c|c|c|}
\hline Serotipo & Susceptible & $\begin{array}{l}\text { Susceptibilidad } \\
\text { intermedia }\end{array}$ & Resistente \\
\hline 1 & 74 & 5 & 0 \\
\hline 3 & 26 & 1 & 0 \\
\hline 4 & 2 & 0 & 0 \\
\hline 5 & 78 & 3 & 0 \\
\hline $6^{a}$ & 4 & 0 & 0 \\
\hline $6 B$ & 11 & 3 & 0 \\
\hline 7 & 1 & 0 & 0 \\
\hline $7 F$ & 16 & 2 & 0 \\
\hline 8 & 3 & 0 & 0 \\
\hline $9 \mathrm{~V}$ & 16 & 1 & 0 \\
\hline $9 N$ & 2 & 0 & 0 \\
\hline $10 \mathrm{~A}$ & 1 & 0 & 0 \\
\hline $12^{*}$ & 2 & 1 & 0 \\
\hline 14 & 8 & 80 & 37 \\
\hline 15 & 1 & 0 & 0 \\
\hline $18 B$ & 1 & 1 & 0 \\
\hline $18 \mathrm{C}$ & 1 & 0 & 0 \\
\hline $19 \mathrm{~A}$ & 8 & 5 & 0 \\
\hline $19 F$ & 4 & 0 & 0 \\
\hline 22 & 1 & 0 & 0 \\
\hline $23 B$ & 0 & 1 & 0 \\
\hline $23 \mathrm{~F}$ & 1 & 0 & 0 \\
\hline $33^{*}$ & 1 & 0 & 0 \\
\hline 34 & 1 & 0 & 0 \\
\hline Total & 263 & 103 & 37 \\
\hline
\end{tabular}


Las 16 cepas de SAMR-AC eran susceptibles a vancomicina, gentamicina y cotrimoxazol; 7 eran resistentes a eritromicina, presentando también resistencia inducible a clindamicina.

\section{Discusión}

En los últimos siete años, la neumonía bacteriana se ha mantenido como una importante causa de ingreso al HP-CHPR, único hospital pediátrico público de referencia nacional en Uruguay. Este hospital concentra la atención del $2^{\circ}$ y $3^{\text {er }}$ nivel de los niños usuarios del Ministerio de Salud Pública residentes en Montevideo y es referente para los niños del sector público del resto del país. De los 332.600 niños que residen en Montevideo, $48 \%$ se asiste en el sector público.

Streptococcus pneumoniae continúa siendo el principal agente de NBAC y su resistencia a los $\beta$-lactámicos no se ha incrementado en este período, evidenciándose una disminución de la resistencia a cefotaxima y en el último año también a penicilina en relación a los datos disponibles de períodos anteriores ${ }^{10}$.

Los serotipos 14, 1, 5 y 3 representan $77 \%$ de los casos confirmados de neumonía neumocóccica, dato a tener en cuenta al momento de decidir la utilización de vacunas para la prevención de la NBAC. Los últimos datos publicados de OPS/SIREVA correspondientes al período 1994-2000 señalaban que los serotipos más frecuentes en infecciones neumocóccicas invasoras fueron por orden de frecuencia: 14, 6A/B, 5, 1, 23F, 19F, 18C, 19A, 9V, 7F, 3, 9N. Estos 13 serotipos representan el $86,1 \%$ de los aislados y no se observaron importantes cambios durante los seis años de vigilancia ${ }^{14}$. En Chile, en un estudio de niños con neumonía bacteriémica y neumonía con efusión, los nueve serotipos de $S$. pneumoniae más frecuentemente asociado a neumonía fueron 14, 6B, 23F, 7B, 1,9V, 5, 18C, 19B ${ }^{15}$. En Uruguay, los datos publicados en los últimos años muestran que $70 \%$ de las cepas de S. pneumoniae aisladas de niños con neumonía corresponden a los serotipos 14,5 y 1 , porcentaje que se mantuvo en el período analizado en este estudio ${ }^{3,10,14}$.

Otro hallazgo relevante es la asociación de algunos serotipos con la presencia de empiema. Se ha descrito la asociación de los serotipos 1 y 3 con esta complicación ${ }^{10,16}$. En nuestra serie, además de estos dos serotipos, el serotipo 5 mostró una asociación significativa con empiema. Para establecer la significación clínica de este hallazgo se requieren estudios complementarios que incorporen el análisis de otros factores potencialmente predisponentes al desarrollo de empiema. Estudios anteriores en nuestro medio señalan una asociación significativa entre el retardo en el diagnóstico

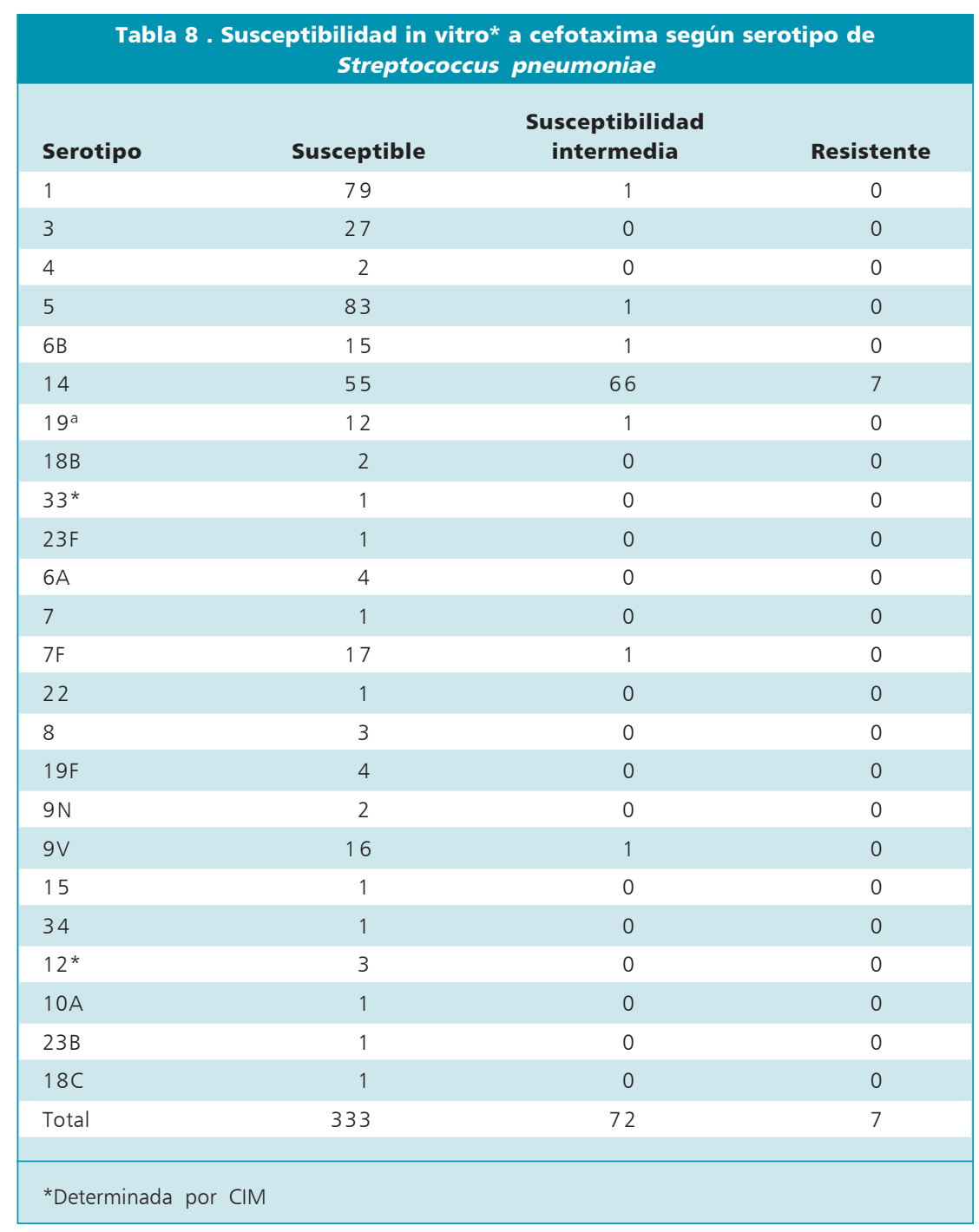

y la presencia de empiema ${ }^{17}$. En otra serie no se observó asociación entre empiema y presencia de cardiopatía congénita, enfermedad neurológica con repercusión sobre la función respiratoria, síndrome de Down, fibrosis quística, ni inmunodeficiencia ${ }^{3}$.

La disponibilidad de vacunas conjugadas que incluyan además del serotipo 14 , el más frecuente, a los serotipos 1, 5 y 3, será de fundamental importancia para contribuir a controlar el problema de la morbimortalidad por neumonía en esta población.

La disponibilidad de una vacuna 9 valente: serotipos $4,6 \mathrm{~B}, 9 \mathrm{~V}, 14,18 \mathrm{C}, 19 \mathrm{~F}, 23 \mathrm{~F}, 1$ y 5 , otorgaría una cobertura para $80 \%$ de los casos de neumonía observados durante el período analizado. Una vacuna 11 valente que agregara los serotipos 3 y 7F tendría una cobertu- 
ra de $91 \%$, frente a la baja cobertura (de $41 \%$ ) que teóricamente otorgaría la vacuna 7 valente hoy disponible. Para estos cálculos no se han tenido en cuenta los cruces antigénicos.

En América Latina, un informe sobre 4.105 aislados de $S$. pneumoniae mostró susceptibilidad disminuida a penicilina en $28,8 \%$. De este grupo, $36,6 \%$ tuvo una $\mathrm{CIM} \geq 2,0 \mathrm{~g} / \mu \mathrm{l}$. Los serotipos con mayor resistencia fueron el 14, que predominó en toda la región, y el 23F que predominó en México. Se detectó reducida susceptibilidad a cefalosporinas de tercera generación en $12,1 \%$. México tuvo la mayor proporción de resistencia a penicilina, $51,6 \%$, con $22 \%$ de resistencia a cefalosporinas de tercera generación. En Brasil las cifras de susceptibilidad disminuida fueron más bajos $(20,9 \%)$, la mayoría de nivel intermedio (CIM 0,12-1,0 $\mu \mathrm{g} / \mathrm{ml})^{14,18}$.

En Uruguay, la resistencia a los $\beta$-lactámicos no ha aumentado en los últimos 7 años. La resistencia intermedia y alta se concentra en el serotipo 14 como fue señalado en publicaciones anteriores ${ }^{10,18}$. Estos resultados estimulan a continuar utilizando guías de tratamiento para las infecciones respiratorias que restrinjan el uso de cefalosporinas de tercera generación, como las que se utilizan desde 1997 en nuestro país ${ }^{1}$. Es probable que el uso racional de antimicrobianos haya contribuido en el control del incremento de la resistencia de $S$. pneumoniae a $\beta$-lactámicos, observada en la década del 90. En todo el período la resistencia intermedia (CIM de $0,12-1,0 \mu \mathrm{g} / \mathrm{ml}$ ) es la que predomina $(25,6 \%)$, las cepas con $\mathrm{CIM} \geq 2,0 \mu \mathrm{g} / \mathrm{ml}$ representaron el $9 \%$.

En nuestro país se dispone de suficiente información para asegurar que el tratamiento de niños infectados con cepas con susceptibilidad disminuida a penicilina no es un problema clínico relevante. Un estudio de niños con empiema tratados con altas dosis de ampicilina o penicilina administradas por vía endovenosa en 6 dosis diarias demostró que las concentraciones de estos antibacterianos en sangre y pleura superan ampliamente la CIM de $4,0 \mu \mathrm{g} / \mathrm{ml}$ durante el $40 \%$ del intervalo interdosis ${ }^{19}$.

La presencia de neumonía por SAMR-AC en nuestro país plantea un nuevo problema diagnóstico y terapéutico. Las cepas que determinan neumonía estafilocóccica en la población estudiada son casi exclusivamente resistentes a meticilina. La gravedad de la enfermedad y las dificultades que ofrece para diferenciarla inicialmente de la neumonía neumocóccica, obligan a poner especial atención a la presentación clínico-radiológica y a la evolución. Es necesario, además, contar con un laboratorio de microbiología preparado para el correcto diagnóstico y la rápida comunicación de los resultados.
Deberán estudiarse las características moleculares de las cepas en la población pediátrica como se lo está realizando en Uruguay en las cepas provenientes de infecciones en adultos. Es necesario continuar estudiando los mecanismos responsables de la emergencia de esta bacteria como agente de infección en la comunidad. Los resultados de estos estudios quizás colaboren en la planificación de medidas de control de la diseminación de esta infección en la comunidad y en los hospitales ${ }^{20}$. En E.U.A. también se ha registrado un aumento de los casos de neumonía con empiema por SAMR- AC a partir del año 2000 21,22.

\section{Conclusiones}

- Streptococcus pneumoniae continúa siendo el agente más frecuente de neumonía bacteriana.

- Las cepas de S. pneumoniae con CIM $\geq 2,0 \mu \mathrm{g} / \mathrm{ml}$ para penicilina $(9 \%)$ y cefotaxima $(1,6 \%)$ no han aumentado en los últimos 7 años.

- El serotipo 14 es el más frecuente y da cuenta de las cepas con $\mathrm{CIM} \leq 2,0 \mu \mathrm{g} / \mathrm{ml}$.

- Las vacunas anti-neumocóccicas disponibles no resultan adecuadas para solucionar el problema de la neumonía grave en esta población.

- La aparición del SAMR-AC como agente de neumonía plantea un nuevo problema clínico terapéutico.

- Es necesario mantener la vigilancia epidemiológica e identificar indicadores que orienten a $S$. aureus como agente etiológico.

\section{Resumen}

La neumonía bacteriana adquirida en la comunidad representa en promedio $8,7 \%$ de los egresos de niños bajo 15 años de edad asistidos en el Hospital Pediátrico del Centro Hospitalario Pereira Rossell; desde 1997 se aplican guías para su diagnóstico y tratamiento. Se estudió la evolución de la etiología de esta enfermedad en los niños hospitalizados en dicho hospital en el período 1998-2004. Streptococcus pneumoniae fue el agente más frecuente todos los años, 91,4 $\pm 2,3 \%$. Su susceptibilidad a penicilina permaneció sin cambios; los serotipos más frecuentes fueron: 14, 1 y 5 . En el año 2002 se identificó el primer caso de Staphylococcus aureus resistente a meticilina adquirido en comunidad; este agente representó $5,8 \%$ de los aislados en 2003 y $6,3 \%$ en 2004 . Es necesario mantener la vigilancia de la etiología de la neumonía bacteriana adquirida en la comunidad para adecuar las guías de diagnóstico y tratamiento a los cambios en los agentes y su susceptibilidad a los antimicrobianos. 


\section{Referencias}

1.- Pírez M C, Martínez O, Ferrari A M, Nairac A, Montano A, Rubio I, et al. Standard case management of pneumonia in hospitalized children in Uruguay, 19971998. Pediatr Infect Dis J 2001; 20: 283-9.

2.- Ferrari A M, Pírez M C, Rubio I, Montano A, Lojo R, Palomino G, et al. Estrategia de atención de niños hospitalizados por infecciones respiratorias agudas bajas. Rev Saúde Pública 2002; 36: 291-300.

3.- Pírez M C, Berrondo C, Giacometti M, Demiguel M, Pascale I, Algorta G, et al. Neumonía bacteriana adquirida en la comunidad en niños hospitalizados. Arch Pediatr Uruguay 2003; 74: 6-14.

4.- Montano A, Algorta G, Pírez M C, Farcilli R, Pascale G, Ferrari A M. Enfermedades invasivas por Haemophilus influenzae tipo b. Impacto de la vacunación en los niños que ingresan al Centro Hospitalario Pereira Rossell. Rev Med Uruguay 2001; 17: 166-70.

5.- Ruocco G, Curto S, Sabio M, Laurani H, Frocht R. Vacunación contra Haemophilus influenzae tipo b en el Uruguay: experiencia e impacto. Rev Panam Salud Pública 1999; 5 (3): 197 - 99.

6.- Farcilli R, Pírez C, Algorta G, Montano A, Ferrari A M. Enfermedades invasivas por Haemophilus influenzae tipo b en los niños que ingresan al Centro Hospitalario Pereira Rossell: 10 años de vacunación. XI Congreso Latinoamericano de Infectología Pediátrica, 8 al 11 de junio de 2005 Veracruz, México.

7.- Algorta G. Staphylococcus aureus meticilino resistente adquirido en la comunidad: Métodos de estudio de la sensibilidad. June 6, 2004. http://www.smu.org.uy/emc/novedades/samr/index.html\#foro. (Accedido 7 diciembre 2004).

8.- Black S, Shinefield H, Baxter R, Austrian R, Bracken L, Hansen J, et al. Postlicensure surveillance for pneumococcal invasive disease after use of heptavalent pneumococcal conjugate vaccine in Northern California Kaiser Permanente. Pediatr Infect Dis J 2004; 23: 485-9.

9.- Kaplan S L, Mason E O, Wald E R, Schutze G E, Bradley J S, Tan T Q, et al. Decrease of invasive pneumococcal infections in children among 8 children's hospitals in the United States after the introduction of the 7-valent pneumococal conjugate disease. Pediatrics 2004; 113 : 443-9.

10. - Hortal M, Camou T, Palacio R, Pérez G, Di Fabio J L y el grupo OPS/SIREVAUruguay. Vigilancia de las neumococcias del niño hospitalizado: su prevención específica (1994-2000). Rev Med Uruguay 2002; 18: 66-75.

11.- Ferrari A M, Nairac A, Peluffo L, Bello O. Neumonía bacteriana en: Atención Pediátrica. Pautas de diagnóstico, tratamiento y Prevención 5ta Edición. Editorial Oficina del Libro, Montevideo 2005.

12.- Isenberg H D. Clinical Microbiology Procedures Handbook. Washington: American Society of Microbiology, 1994.

13.- National Committee for Clinical Laboratory Standards. Performance Standards for antimicrobial susceptibility testing; 14th Informational Supplement NCCLS Document M100-S14. Wayne, PA: National Committee for Clinical Laboratory Standards, 2004.

14.- Di Fabio J L, Castañeda E, Agudelo CI, De la Hoz F, Hortal M, Camou T, et al. Evolution of Streptococcus pneumoniae serotypes and penicillin susceptibility in Latin-American, SIREVA Vigia Group. 1993 to 1999. PAHO SIREVA Vigia Study Group. Pan American Health Organization. Pediatr Infect Dis J 2001; 20: 959-67.

15.- Lagos R, Muñoz A, Valenzuela M T, Heitmann I, Levine M. Population based surveillance for hospitalized and ambulatory pediatric invasive pneumococcal diseases in Santiago, Chile. Pediatr Infect Dis J 2002; 21: 1115-23.

16.- Tan T Q, Mason E O, Wald E R, Barson W J, Schutze G E, Bradley J S, et al. Clinical characteristics of children with complicated pneumonia caused by Streptococcus pneumoniae. Pediatrics 2002; 110: 1-6.

17.- Gutiérrez S, Stewart J, De Olivera N, Gandaro P, García C, Pírez M C, et al. Factores de riesgo de empiema pleural en niños uruguayos menores de 5 años. Rev Chil Pediatr 2004; 75: 536-42.

18.- Hortal M, Lovgren M, de la $\mathrm{Hoz}$ F, Agudelo C I, Brandileone MC, Camou T, et al. Antibiotic resistance in Streptococcus pneumoniae in six Latin-American countries. Microb Drug Resist 2001; 7: 391401.

19.- Giachetto G, Pírez M C, Nanni L, Martínez A, Montano A, Kaplan S L, et al. Ampicillin and penicillin concentration in serum and pleura fluid of hospitalized children with community acquired pneumoniae. Pediatric Infect Dis J 2004; 23 : 625-9.

20.- Ma X X, Galiana A, Pedreira W, Mowszowicz M, Christophersen I, Machiavello S, et al. Community acquired methicillin-resistant Staphylococcus aureus, Uruguay. Emerg Infect Dis 2005; 11: 973-6.

21.- Mishaan A M, Mason E O Jr, MartínezAguilar G, Hammerman W, Propts J J, Lupski J R, et al. Emergence of a predominant clone of community-acquired Staphylococcus aureus among children in Houston, Texas. Pediatr Infect Dis J 2005; 24: 201-6.

22.- Schultz K D, Fan L L, Pinsky J, Ochoa L, Smith E O, Kaplan S L, et al. The changing face of pleural empyemas in children: epidemiology and management. Pediatrics 2004; 113: 1735-40. 